\title{
Kink and anti-kink wave solutions for the generalized KdV equation with Fisher-type nonlinearity
}

\author{
Hüseyin Koçak ${ }^{*}$ \\ Quantitative Methods Division, Pamukkale University, 20160, Denizli, Turkey \\ hkocak@pau.edu.tr
}

\section{ARTICLE INFO}

Article history:

Received: 20 April 2020

Accepted: 10 November 2020

Available Online: 2 April 2021

Keywords:

The $g K d V$-Fisher equation

Dispersion-convection-reaction model

Travelling wave solutions

AMS Classification 2010:

35C07, 76B15, 76V05

\section{ABSTRACT}

This paper proposes a new dispersion-convection-reaction model, which is called the gKdV-Fisher equation, to obtain the travelling wave solutions by using the Riccati equation method. The proposed equation is a third-order dispersive partial differential equation combining the purely nonlinear convective term with the purely nonlinear reactive term. The obtained global and blow-up solutions, which might be used in the further numerical and analytical analyses of such models, are illustrated with suitable parameters.

\section{Introduction}

This study focuses on the travelling wave solutions of a newly introduced dispersion-convection-reaction model

$u_{t}+\varepsilon u^{n} u_{x}+\mu u_{x x x}=r u\left(1-u^{n}\right)$,

where $n>0, \varepsilon$ is a parameter for the purely nonlinear convection term, $\mu$ is a parameter for the linear dispersion term and $r$ is a parameter for the purely nonlinear reaction term. One can easily obtain the talented equations, such as the generalized $\mathrm{KdV}$ $(\mathrm{gKdV})$ equation [1-9] and the dispersive-Fisher equation [10] by taking $r=0$ and $\varepsilon=0$ in Eq. (1), respectively. There have been many cooperative combinations of dispersion with the different terms, such as dissipation, convection, diffusion and reaction [11-15]. Here, by combining the gKdV equation [2-8] with the Fisher-type (or KPP-type) nonlinearity [1623], we call Eq. (1) as the gKdV-Fisher equation.

Recently, Galaktionov has focused on the higher-order versions of the KPP (or Fisher) type problem in the parabolic, dispersive and hyperbolic equations, see [2123] for fruitful discussions. Fortunately, the third-order dispersive partial differential equation including the Fisher-type nonlinearity

$u_{t}=u_{x x x}+u(1-u)$,

with two travelling wave solutions

${ }^{*}$ Corresponding author

$$
\begin{aligned}
u_{1}(x, t)= & \frac{1}{2}-\frac{9}{4} \tanh \left(\frac{19}{60} t-\frac{1}{60} 9900^{1 / 3}\left(x+\xi_{0}\right)\right) \\
& +\frac{11}{4} \tanh ^{3}\left(\frac{19}{60} t-\frac{1}{60} 9900^{1 / 3}\left(x+\xi_{0}\right)\right), \\
u_{2}(x, t)= & \frac{1}{2}+\frac{3}{4} \tanh \left(\frac{19}{60} t+\frac{1}{60} 30^{2 / 3}\left(x+\xi_{0}\right)\right) \\
& -\frac{1}{4} \tanh ^{3}\left(\frac{19}{60} t+\frac{1}{60} 30^{2 / 3}\left(x+\xi_{0}\right)\right),
\end{aligned}
$$

has been proposed in [10]. More recently, the exact travelling waves of the KdV-Burgers-Fisher equation

$u_{t}+\varepsilon u u_{x}-v u_{x x}+\mu u_{x x x}=r u(1-u)$,

have been investigated in [24].

We would also like to remind the neighbouring nonlinear parabolic equation, which is a diffusionconvection-reaction model and called the generalized Burgers-Fisher equation [25,26],

$u_{t}+\varepsilon u^{n} u_{x}-v u_{x x}=r u\left(1-u^{n}\right)$,

with the exact solution

$u(x, t)=\left(\frac{\tanh (\xi)+1}{2}\right)^{\frac{1}{n}}$,

where

$$
\xi=-\frac{n \varepsilon}{2 v(n+1)} x+\frac{n\left(\varepsilon^{2}+v r(n+1)^{2}\right)}{2 v(n+1)^{2}} t+\xi_{0} .
$$


In the next section, we use the Riccati equation method [27-36] to reveal the travelling wave solutions of the gKdV-Fisher equation, which are the cooperative results of the proposed combined model. Here, it is reasonable to expect kink and antikink wave solutions because of the reaction term in the proposed equation.

\section{Method and application}

Let us first take the wave variable $\xi=k x+w t+\xi_{0}$ and $u(x, t)=u(\xi)$ in Eq. (1) to obtain the reduced nonlinear ODE as

$w u^{\prime}+\varepsilon k u^{n} u^{\prime}+\mu k^{3} u^{\prime \prime \prime}-r u\left(1-u^{n}\right)=0$.

The solution of Eq. (5) is assumed to be expressed as

$u=\sum_{i=0}^{M} a_{i} z^{i}$

where the parameters, $a_{0}, \ldots, a_{M}$ and $M$, can be determined later and $z=z(\xi)$ is the solution of the following classical Riccati equation [27-35]:

$z^{\prime}=1-z^{2}$

which has the forms

$z=\tanh (\xi)$ and $z=\operatorname{coth}(\xi)$.

Here, using the advantage of the Riccati equation, higher-order derivatives of Eq. (7) can be obtained as

$z^{\prime \prime}=-2 z+2 z^{3}$,

$z^{\prime \prime \prime}=-2+8 z^{2}-6 z^{4}$.

If we next substitute Eq. (6) with Eqs. (7), (9) and (10) into Eq. (5) and balance $z^{\prime \prime \prime}$ with $z^{n} z^{\prime}$, we have

$M+3=n M+M+1$ resulting in $M=\frac{2}{n}$.

In order to obtain the positive integer $M$ values for Eq. (6), we use the transformation

$u=v^{\frac{2}{n}}$

in Eq. (5), which yields

$$
\begin{aligned}
& 2 w n^{2} v^{2} v^{\prime}+2 \varepsilon k n^{2} v^{4} v^{\prime}+2 \mu k^{3} n^{2} v^{2} v^{\prime \prime \prime}-6 \mu k^{3} n^{2} v v^{\prime} v^{\prime \prime} \\
& +12 \mu k^{3} n v v^{\prime} v^{\prime \prime}+4 \mu k^{3} n^{2}\left(v^{\prime}\right)^{3}-12 \mu k^{3} n\left(v^{\prime}\right)^{3} \\
& -r n^{3} v^{3}+r n^{3} v^{5}=0 .
\end{aligned}
$$

If we now apply the same procedure by using the expression for $v$ as

$v=\sum_{i=0}^{M} a_{i} z^{i}$

in Eq. (13) with Eqs. (7), (9) and (10), and balancing the highest power of $z$, we have $M=1$, which yields the solution of Eq. (13) to be in the form

$v=a_{0}+a_{1} z$.

Let us next use Eq. (15) in Eq. (13) and collect the coefficients for the same powers of $z$ as follows:

$$
\begin{aligned}
z^{0}: & 4 a_{1}^{3} k^{3} \mu n^{2}-4 k^{3} \mu n^{2} a_{0}^{2} a_{1}+2 k n^{2} \varepsilon a_{0}^{4} a_{1}+n^{3} r a_{0}^{5} \\
& -12 a_{1}^{3} k^{3} \mu n+8 a_{1}^{3} k^{3} \mu-n^{3} r a_{0}^{3}+2 n^{2} w a_{0}^{2} a_{1}=0, \\
z^{1}: & 4 k^{3} \mu n^{2} a_{0} a_{1}^{2}+8 k n^{2} \varepsilon a_{0}^{3} a_{1}^{2}+5 n^{3} r a_{0}^{4} a_{1}+4 n^{2} w a_{0} a_{1}^{2} \\
& -24 k^{3} \mu n a_{0} a_{1}^{2}-3 n^{3} r a_{0}^{2} a_{1}=0, \\
z^{2}: & 16 k^{3} \mu n^{2} a_{0}^{2} a_{1}-4 a_{1}^{3} k^{3} \mu n^{2}-2 k n^{2} \varepsilon a_{0}^{4} a_{1}-24 a_{1}^{3} k^{3} \mu \\
& +12 k n^{2} \varepsilon a_{0}^{2} a_{1}^{3}+10 n^{3} r a_{0}^{3} a_{1}^{2}+12 a_{1}^{3} k^{3} \mu n+2 n^{2} w a_{1}^{3} \\
& -3 n^{3} r a_{0} a_{1}^{2}-2 n^{2} w a_{0}^{2} a_{1}=0, \\
z^{3}: \quad & 8 k^{3} \mu n^{2} a_{0} a_{1}^{2}-8 k n^{2} \varepsilon a_{0}^{3} a_{1}^{2}+8 k n^{2} \varepsilon a_{0} a_{1}^{4}-n^{3} r a_{1}^{3} \\
& +10 n^{3} r a_{0}^{2} a_{1}^{3}+48 k^{3} \mu n a_{0} a_{1}^{2}-4 n^{2} w a_{0} a_{1}^{2}=0, \\
z^{4}: & 4 a_{1}^{3} k^{3} \mu n^{2}-12 k^{3} \mu n^{2} a_{0}^{2} a_{1}-12 k n^{2} \varepsilon a_{0}^{4} a_{1}^{3}-2 n^{2} w a_{1}^{3} \\
& +2 k n^{2} \varepsilon a_{1}^{5}+5 n^{3} r a_{0} a_{1}^{4}+12 a_{1}^{3} k^{3} \mu n+24 a_{1}^{3} k^{3} \mu=0, \\
z^{5}: & n^{3} r a_{1}^{5}-12 k^{3} \mu n^{2} a_{0} a_{1}^{2}-8 k n^{2} \varepsilon a_{0} a_{1}^{4}-24 k^{3} \mu n a_{0} a_{1}^{2}, \\
z^{6}: & -4 a_{1}^{3} k^{3} \mu n^{2}-2 k n^{2} \varepsilon a_{1}^{5}-12 a_{1}^{3} k^{3} \mu n-8 a_{1}^{3} k^{3} \mu=0 .
\end{aligned}
$$

As the final step, solving the nonlinear system (16) for $a_{0}, a_{1}$ and nonzero $k, w, \varepsilon, \mu, r, n$ parameters by using Maple, we have the following solutions for $\varepsilon \mu<0$ :

$u_{1,2}=\left(\frac{\tanh \left(k x+w t+\xi_{0}\right) \pm 1}{2}\right)^{\frac{2}{n}}$

and

$u_{3,4}=\left(\frac{\operatorname{coth}\left(k x+w t+\xi_{0}\right) \pm 1}{2}\right)^{\frac{2}{n}}$,

where

$r=\mp \frac{\varepsilon(n+4) \sqrt{-2 \varepsilon \mu(n+1)(n+2)}}{2 \mu(n+1)^{2}(n+2)}$,

$k= \pm \frac{r(n+1) n}{2 \varepsilon(n+4)}$,

$w= \pm \frac{r\left(n^{2}+6 n+12\right) n}{4(n+2)(n+4)}$

Figure 1 exhibits the long-time behaviour for the global solution $u_{1}(x, t)$ of the $\mathrm{gKdV}$-Fisher equation in Eq. (17) for the different values of the parameters, which represent kink and antikink waves. One can easily see that the propagations of waves are backward in Figure 1-(b), i.e. $k, w>0$, and forward in Figure 1-(a) and Figure 1-(c). Another global solution $u_{2}(x, t)$ in Eq. (17) is displayed in Figure 2 for the given parameters, which also exhibit kink and antikink waves.

On the other hand, the blow-up solutions $u_{3}(x, t)$ and $u_{4}(x, t)$ in Eq. (18) are displayed in Figure 3 and Figure 4 , respectively. 


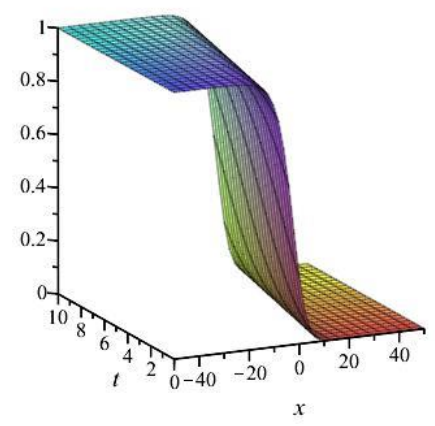

(a) $\varepsilon=-1, \mu=1, r=5 \sqrt{3} / 12, n=1, \xi_{0}=0$.

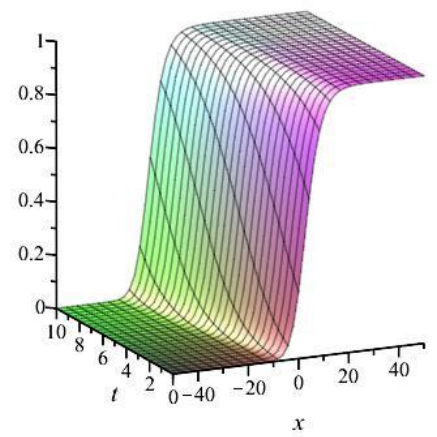

(b) $\varepsilon=1, \mu=-1, r=5 \sqrt{3} / 12, n=1, \xi_{0}=0$.

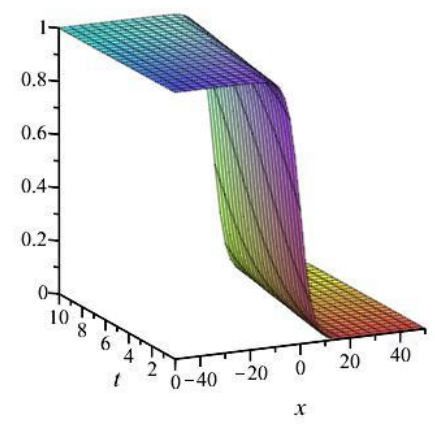

(c) $\varepsilon=-1, \mu=1, r=\sqrt{6} / 6, n=2, \xi_{0}=0$.

Figure 1. The solution $u_{1}(x, t)$ of the gKdV-Fisher equation for the different suitable parameters.

Because of the nature of the complex nonlinear phenomena, it is reasonable to find the blow-up solutions for the mix of the different entities with nonlinearity. Fortunately, we can reveal the cooperative combinations of dispersion, convection and reaction with the parameters in Eq. (19) for the global solutions of the gKdV-Fisher equation, which represent kink and antikink waves, see Figure 1 and 2.

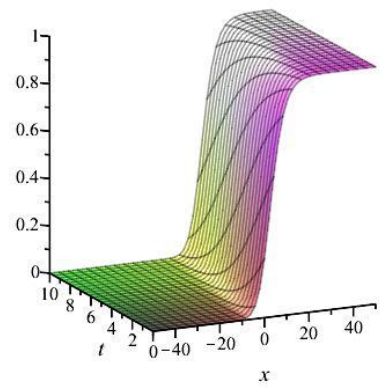

(a) $\varepsilon=-1, \mu=1, r=-5 \sqrt{3} / 12, n=1, \xi_{0}=0$.

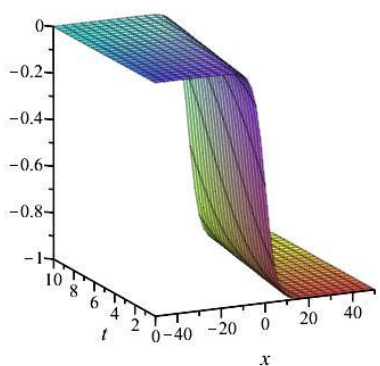

(b) $\varepsilon=-1, \mu=1, r=-\sqrt{6} / 6, n=2, \xi_{0}=0$.

Figure 2. The solution $u_{2}(x, t)$ of the gKdV-Fisher equation for the different suitable parameters.

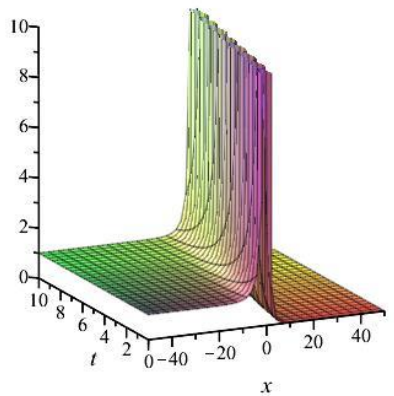

(a) $\varepsilon=-1, \mu=1, r=5 \sqrt{3} / 12, n=1, \xi_{0}=0$.

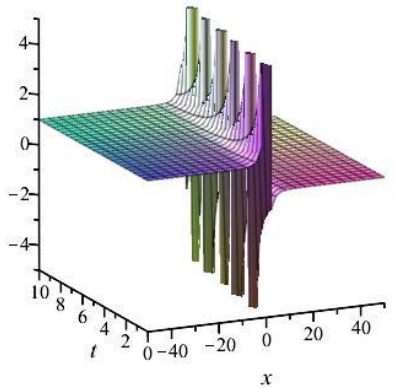

(b) $\varepsilon=-1, \mu=1, r=\sqrt{6} / 6, n=2, \xi_{0}=0$.

Figure 3. The solution $u_{3}(x, t)$ of the gKdV-Fisher equation for the different suitable parameters. 


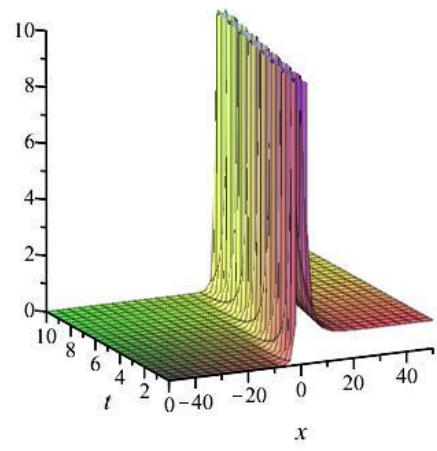

(a) $\varepsilon=-1, \mu=1, r=-5 \sqrt{3} / 12, n=1, \xi_{0}=0$.

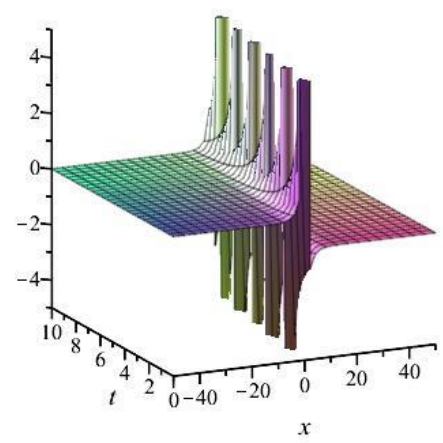

(b) $\varepsilon=-1, \mu=1, r=-\sqrt{6} / 6, n=2, \xi_{0}=0$.

Figure 4. The solution $u_{4}(x, t)$ of the gKdV-Fisher equation for the different suitable parameters.

\section{Conclusion}

A nonlinear dispersion-convection-reaction model, called the $\mathrm{gKdV}$-Fisher equation, has been introduced to investigate the travelling wave solutions. A classical and efficient the Riccati equation method has been used to investigate two new global and two new blow-up solutions. One can easily see that the reaction term in the proposed equation yields kink and antikink wave solutions, which can be used in the other various numerical and analytical investigations on the application of such combined model to scientific problems. Further research would be based on investigating $\mathrm{N}$-soliton solutions of the third and higher odd-order PDEs including Fisher-type nonlinearity.

\section{Acknowledgments}

The author would like to thank Prof. Dr. V. A. Galaktionov for the guidance in the PDE world.

\section{References}

[1] Korteweg, D. J., \& de Vries, G. (1895). On the change of form of long waves advancing in a rectangular canal, and on a new type of long stationary waves. The London, Edinburgh, and Dublin Philosophical Magazine and Journal of Science, 39(240), 422-443.
[2] Tsutsumi, M., Mukasa, T., \& Iino, R. (1970). On the generalized Korteweg-de Vries Equation. Proceedings of the Japan Academy, 46(9), 921-925.

[3] Gardner, C. S., Greene, J. M., Kruskal, M. D., \& Miura, R. M. (1974). Korteweg-devries equation and generalizations. VI. methods for exact solution. Communications on Pure and Applied Mathematics, 27(1), 97-133.

[4] Tagare, S. G., \& Chakrabarti, A. (1974). Solution of a generalized Korteweg-de Vries equation. Physics of Fluids, 17, 1331-1332.

[5] Rosenau, P., \& Hyman, M. (1993). Compactons: Solitons with Finite Wavelength. Physical Review Letters, 70, 564-567.

[6] Colliander, J. E., \& Kenig, C. E. (2002). The generalized Korteweg-de Vries equation on the half line. Communications in Partial Differential Equations, 27, 2187-2266.

[7] Wazwaz, A. M. (2006). Kinks and solitons for the generalized $\mathrm{KdV}$ equation with two power nonlinearities. Applied Mathematics and Computation, 183, 1181-1189.

[8] Tao, T. (2007). Two remarks on the generalised Korteweg de-Vries equation. Discrete \& Continuous Dynamical Systems - A, 18, 1-14.

[9] Tao, T. (2008). Global behaviour of nonlinear dispersive and wave equations. Current Developments in Mathematics, 2006, 255-340.

[10] Pinar, Z., \& Koçak, H. (2018). Exact solutions for the third-order dispersive-Fisher equations. Nonlinear Dynamics, 91(1), 421-426.

[11] Rosenau, P. (1998). On a class of nonlinear dispersive-dissipative interactions. Physica D: Nonlinear Phenomena, 123(1-4), 525-546.

[12] Wazwaz, A. M. (2006). The tanh method for compact and noncompact solutions for variants of the KdV-Burger and the $\mathrm{K}(\mathrm{n}, \mathrm{n})$-Burger equations. Physica D: Nonlinear Phenomena, 213, 147-151.

[13] Galaktionov, V. A., Miditieri, E. L., \& Pohozaev, S. I. (2014). Blow-up for higher-order parabolic, hyperbolic, dispersion and Schrödinger equations. Monographs and Research Notes in Mathematics, Chapman and Hall/CRC, Boca Raton.

[14] Koçak, H. (2017). Similarity solutions of nonlinear third-order dispersive PDEs: The first critical exponent. Applied Mathematics Letters, 74, 108113.

[15] Koçak, H., \& Pinar Z. (2018). On solutions of the fifth-order dispersive equations with porous medium type non-linearity. Waves in Random and Complex Media, 28(3), 516-522.

[16] Fisher, R. A. (1937). The wave of advance of advantageous genes. Annals of Eugenics, 7(4), 355369.

[17] Kolmogorov, A. N., Petrovskii, I. G., \& Piskunov, 
N. S. (1937). Study of the diffusion equation with growth of the quantity of matter and its application to a biological problem. Byull. Moskov. Gos. Univ., Sect. A, 1, 1-26. (English. transl. In: Dynamics of Curved Fronts, P. Pelce, Ed., Acad. Press, Inc., New York, 1988, 105-130.)

[18] Ablowitz, M. J., \& Zeppetella, A. (1979). Explicit solutions of Fisher's equation for a special wave speed. Bulletin of Mathematical Biology, 41, 835840 .

[19] Wazwaz, A. M. (2008). Analytic study on Burgers, Fisher, Huxley equations and combined forms of these equations. Applied Mathematics and Computation, 195(2), 754-761.

[20] Gilding, B. H., \& Kersner, R. (2012). Travelling waves in nonlinear diffusion-convection-reaction. Vol 60, Birkhäuser Basel.

[21] Galaktionov, V. A. (2012). Towards the KPPProblem and $\log$ t-Front Shift for Higher-Order Nonlinear PDES II. Quasilinear Bi-and TriHarmonic Equations. arXiv:1210.5063.

[22] Galaktionov, V. A. (2012). Towards the KPP-Problem and $\log$ t-Front Shift for Higher-Order Nonlinear PDEs III. Dispersion and Hyperbolic Equations. arXiv: 1210.5084.

[23] Galaktionov, V. A. (2013). The KPP-Problem and $\log$ t-Front Shift for Higher-Order Semilinear Parabolic Equations. Proceedings of the Steklov Institute of Mathematics, 283, 44-74.

[24] Koçak, H. (2020). Travelling Waves in Nonlinear Media with Dispersion, Dissipation and Reaction. Chaos: An Interdisciplinary Journal of Nonlinear Science, 30(9), 093143.

[25] Chen, H., \& Zhang, H. (2004). New multiple soliton solutions to the general Burgers-Fisher equation and the Kuramato-Sivashinsky equation. Chaos, Soltions and Fractals, 19, 71-76.

[26] Wazwaz, A. M. (2005). The tanh method for generalized forms of nonlinear heat conduction and Burgers-Fisher equations. Applied Mathematics and Computation, 169, 321-338.

[27] Huibin, L., \& Kelin, W. (1990). Exact solutions for nonlinear equations. I. Journal of Physics A: Mathematical and General, 23(17), 3923-3928.

[28] Malfliet, W. (1992). Solitary wave solutions of nonlinear wave equations. American Journal of Physics, 60(7), 650-654.

[29] Malfliet, W., \& Hereman, W. (1996). The Tanh Method: I. Exact Solutions of Nonlinear Evolution and Wave Equations. Physica Scripta, 54, 563-568.

[30] Fan, E. G. (2002). Traveling Wave Solutions for Nonlinear Equations Using Symbolic Computation. Computers and Mathematics with Applications, 43, 671-680.

[31] Kudryashov, N. A. (2005). Simplest equation method to look for exact solutions of nonlinear differential equations. Chaos, Solitons \& Fractals, 24 (5), 1217-1231.

[32] Kudryashov, N. A. (2009). Seven common errors in finding exact solutions of nonlinear differential equations. Communications in Nonlinear Science and Numerical Simulation, 14, 3507-3529.

[33] Wazwaz, A. M. (2009). Partial Differential Equations and Solitary Wave Theory. Higher Education Press, Beijing and Springer-Verlag Berlin Heidelberg.

[34] Griffiths, G. W., \& Schiesser, W. E. (2011). Traveling Wave analysis of Partial Differential Equations: Numerical and Analytical Methods with MATLAB and MAPLE. Academic Press, New York.

[35] Polyanin, A. D., \& Zaitsev, V. F. (2012). Handbook of Nonlinear Partial Differential Equations. Chapman and Hall/CRC, Boca Raton.

[36] Manafian, J., \& Lakestani, M. (2017). A new analytical approach to solve some of the fractionalorder partial differential equations. Indian Journal of Physics, 91, 243-258.

Hüseyin Koçak obtained his PhD degree in Mathematical Sciences from the University of Bath, UK in 2015. He has been working as Asst. Prof. of Quantitative Methods at the Pamukkale University since 2016.

http://orcid.org/0000-0001-9683-6096

An International Journal of Optimization and Control: Theories \& Applications (http://ijocta.balikesir.edu.tr)

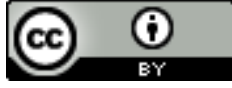

This work is licensed under a Creative Commons Attribution 4.0 International License. The authors retain ownership of the copyright for their article, but they allow anyone to download, reuse, reprint, modify, distribute, and/or copy articles in IJOCTA, so long as the original authors and source are credited. To see the complete license contents, please visit http://creativecommons.org/licenses/by/4.0/. 